\title{
African signatures of recent positive selection in human FOXI1
}

\author{
Andrés Moreno-Estrada ${ }^{1,3}$, Estel Aparicio-Prat ${ }^{1}$, Martin Sikora', Johannes Engelken ${ }^{1}$, Anna Ramírez-Soriano', \\ Francesc Calafell ${ }^{1,2}$, Elena Bosch ${ }^{1,2^{*}}$
}

\begin{abstract}
Background: The human FOXI1 gene codes for a transcription factor involved in the physiology of the inner ear, testis, and kidney. Using three interspecies comparisons, it has been suggested that this may be a gene under human-specific selection. We sought to confirm this finding by using an extended set of orthologous sequences. Additionally, we explored for signals of natural selection within humans by sequencing the gene in 20 Europeans, 20 East Asians and 20 Yorubas and by analysing SNP variation in a $2 \mathrm{Mb}$ region centered on FOXI1 in 39 worldwide human populations from the HGDP-CEPH diversity panel.
\end{abstract}

Results: The genome sequences recently available from other primate and non-primate species showed that FOXI1 divergence patterns are compatible with neutral evolution. Sequence-based neutrality tests were not significant in Europeans, East Asians or Yorubas. However, the Long Range Haplotype (LRH) test, as well as the iHS and XP-Rsb statistics revealed significantly extended tracks of homozygosity around FOXI1 in Africa, suggesting a recent episode of positive selection acting on this gene. A functionally relevant SNP, as well as several SNPs either on the putatively selected core haplotypes or with significant iHS or XP-Rsb values, displayed allele frequencies strongly correlated with the absolute geographical latitude of the populations sampled.

Conclusions: We present evidence for recent positive selection in the FOXI1 gene region in Africa. Climate might be related to this recent adaptive event in humans. Of the multiple functions of FOXI1, its role in kidney-mediated water-electrolyte homeostasis is the most obvious candidate for explaining a climate-related adaptation.

\section{Background}

FOXI1 is a family member of the forkhead-box (FOX) transcription factors that are characterized by the FOX $\sim 100$ amino acid monomeric DNA-binding domain [1]. Different mutations in the human FOXI1 gene and its regulatory binding site on $S L C 26 A 4$ (also known as pendrin) have been shown to compromise the transcription of this anion transporter gene in patients with Pendred Syndrome and nonsyndromic enlargement of the vestibular aqueduct [2]. Foxi1 has also been recognised as a key factor necessary for correct patterning of distal nephron epithelium and adequate acid-base homeostasis in the kidney causing distal renal tubular acidosis in Foxi1 $^{-1-}$ mice [3]. Moreover, Foxi1 has been reported to

\footnotetext{
* Correspondence: elena.bosch@upf.edu

'Institut de Biologia Evolutiva (UPF-CSIC), Departament de Ciències Experimentals i de la Salut, Universitat Pompeu Fabra, Parc de Recerca Biomèdica de Barcelona, C/Dr. Aiguader 88, 08003 Barcelona, Spain Full list of author information is available at the end of the article
}

be a crucial activator of the B1-subunit of the vacuolar $\mathrm{H}+$-ATPase proton pump (ATP6V1B1) as well as for pendrin (encoded by $S L C 26 A 4$ ) and carbonic anhydrase II expression in the epididymal cells, which are required for a correct mouse sperm maturation [4]. Overall, these findings have led to the hypothesis that mutations in the human FOXI1 gene might cause sensorineural deafness with distal renal tubular acidosis and male infertility [4].

Thus, FOXI1 seems to be involved in the acid-base balance of at least three different organs: inner ear, testis, and kidney. Using human-chimpanzee-mouse orthologous gene trios, FOXI1 was suggested as an example of a gene involved in hearing that appeared to present a subset of sites with accelerated amino acid substitution in the human lineage [5]. However, the availability of additional FOXI orthologous sequences from a variety of species, including other primates, has permitted this initial finding to be revised. Genes involved in sensory perception show accelerated evolution or are under
C Biomed Central 
positive selection in the human lineage [5,6]. Reproduction was only found as a biological function showing an excess of positively selected genes when comparing human and chimpanzee, and thus it could not be established in which of the two branches positive selection acted [7]. Within humans, adaptation to different climates may have entailed adapting kidney function to varying hydration levels. For instance, a thrifty genotype in water retention is suggested to have been selected in the gruelling trans-Atlantic voyage of the slave ships, resulting in a higher prevalence of hypertension in African-Americans [8]. Moreover, variants influencing salt homeostasis in the CYP3A5 and AGT loci have been shown to be targets of a selective pressure varying in intensity in correlation with latitude [9]. It is of great interest, therefore, to investigate whether FOX1I has been the target of local adaptation in humans. In order to test such a hypothesis, we evaluated the patterns of nucleotide variation and looked for signals of positive natural selection in the human FOXI1 gene by resequencing 20 Europeans, 20 Asians and 20 Yorubas and by assaying SNP variation in 971 individuals from the Human Genome Diversity Panel (HGDP-CEPH Diversity Panel; [10]).

\section{Methods \\ Population samples}

Sixty HapMap DNA samples for resequencing were obtained from Coriell Cell Repositories (Camden, NJ, USA). These consisted of 20 Europeans (Utah residents with ancestry from Northern and Western Europe), 20 Asians (10 Japanese from Tokyo and 10 Chinese from Beijing) and 20 Yorubas (from Ibadan in Nigeria). Coriell Repository numbers for these samples are listed in Table S1 (Additional File 1).

SNP genotypes were obtained for the Human Genome Diversity Panel (HGDP-CEPH), which contains 1,064 DNA samples from individuals representing 51 populations globally distributed [10]. In all statistical analyses atypical, duplicated individuals and deduced first-degree relatives were removed by using the H971 subset recommended by Rosenberg [11]. In order to maximize sample sizes, genotyped samples were re-grouped into 39 populations based on geographic and ethnic criteria as in Gardner et al. [12]. For part of the analysis, populations were further grouped into seven geographical regions: Sub-Saharan Africa (SSAFR), Middle EastNorth Africa (MENA), Europe (EUR), Central-South Asia (CSASIA), East Asia (EASIA), Oceania (OCE) and America (AME).

All samples used were originally collected with proper informed consent. The research protocol was approved by the Clinical Research Ethical Committee of the Municipal Institute of Health Care (CEIC-IMAS).

\section{Resequencing of the FOXI1 gene}

We amplified two overlapping fragments of 2,100 bp and 2,099 bp covering the entire FOXI1 genomic sequence. PCRs were performed in a total volume of $25 \mu \mathrm{l}$, containing $0.2 \mathrm{mM} \mathrm{dNTPs}, 1.5 \mathrm{mM} \mathrm{MgCl}_{2}$, $0.5 \mu \mathrm{M}$ of each primer (Additional File 2, Table S2), $1 \times$ buffer, $0.05 \mathrm{U}$ Taq polymerase (Ecogen), and $10 \mathrm{ng}$ genomic DNA. PCR conditions were as follows: $3 \mathrm{~min}$ at $94^{\circ} \mathrm{C}, 30$ cycles of $94^{\circ} \mathrm{C}$ for $30 \mathrm{sec}, 55^{\circ} \mathrm{C}$ for $30 \mathrm{sec}$ and $72^{\circ} \mathrm{C}$ for $3 \mathrm{~min}$; and a final step of extension of $5 \mathrm{~min}$ at $72^{\circ} \mathrm{C}$. PCR products were purified using MultiScreen PCR $\mu 96$ chemistry (Millipore) according to the manufacturer's protocol. Amplified PCR products were sequenced using Big Dye Terminator chemistry ver.3.1 (Applied Biosystems) and the sequencing primers listed in Table S2 (Additional File 2). Extension products were purified using the Montage SEQ96 Cleanup kit (Millipore) and run on an ABI 3730 XL sequencer. Sequence analysis and contig assembly for each sample were performed with the Seqman module of the DNASTAR Lasergene software ver. 7.1.0 and visually inspected at least twice. All polymorphic sites were checked manually and heterozygote positions were confirmed by reamplifying and resequencing the SNP site from the same or the opposite strand. Nucleotide sequence data reported are available in the GenBank database under accession numbers GU049150 to GU049269.

\section{SNP genotyping data}

A total of 21 SNPs covering $400 \mathrm{~kb}$ centered on the FOXI1 gene region were genotyped on the HGDPCEPH diversity panel. SNPs were selected every 5-10 kb within the gene and up to around $30 \mathrm{~kb}$ in both $5^{\prime}$ and 3 ' end flanking regions; from this point, an extra SNP was then added every $40 \mathrm{~kb}$ up to around $200 \mathrm{~kb}$ in both flanking regions. Preference was given to SNPs with a minor allele frequency (MAF) over 10\%, which were compiled from HapMap (Release 7 May 2004) and dbSNP (Build 121 June 2004) databases. SNPs were typed using the SNPlex Genotyping System from Applied Biosystems within a larger set of SNPs covering additional genes as described elsewhere [13]. Illumina HumanHap650K Beadchip genotypes on the HGDPCEPH panel were downloaded from the Stanford Human Genome Center website. From this publicly available data we extracted a SNP genotype set centered on FOXI1 and extending up to $2 \mathrm{Mb}$ (with a total of 693 SNPs), which was complemented by 15 of our previously genotyped SNPs.

\section{Statistical analysis}

In order to test for positive selection in the human lineage we initially applied the improved branch-site test 2 of positive selection [14] on a phylogeny containing five 
mammalian species (Additional File 3, Figure S1). The human reference sequence for FOXI1 and its orthologous sequences in chimpanzee, mouse, rat and dog had been previously extracted from Ensembl (Gene IDs ENSG00000168269, ENSPTRG00000017514, ENSM USG00000047861, ENSRNOG00000006293, ENSCA FG00000016968) and aligned with ClustalW [15]. The alignment length was $1,137 \mathrm{bp}$, which became $1,110 \mathrm{bp}$ (97.6\%) after removing gaps (Additional File 4, Figure S2). Calculations for the corresponding null and alternative hypotheses were performed using the codeml program implemented in the PAML package [16]. Results for additional likelihood ratio tests of positive selection on the human lineage considering different multispecies alignments were obtained from the Human PAML browser [17]. Table S3 (Additional File 5) summarises the likelihood ratio test results for the branch test (Model $\mathrm{H}$ versus Model $\mathrm{H}$ null) and for the strict branch positive site test of positive selection (Model A versus Model A null).

Arlequin [18] was used to calculate $F_{S T}$ values among the 39 populations studied with a locus by locus Analysis of Molecular Variance (AMOVA) [19]. Haplotypes were inferred from sequencing data using the Bayesian statistical method in the program PHASE 2.1 [20] using the default parameter set with 1,000 iterations. Haplotype estimation from the $400 \mathrm{~kb}$ and $2 \mathrm{Mb}$ SNP genotype data sets was performed with FastPHASE [21]. The Network 4.5.0.1 software package (http://www.fluxusengineering.com) was used to construct the minimum mutation network by means of the median-joining algorithm [22,23]. The ancestral states for the FOXI1 polymorphic positions were inferred from the previously aligned orthologous sequences in chimpanzee, mouse and rat but adding macaque (ENSMMUG00000014124) to the alignment.

Nucleotide diversity statistics and analysis of population polymorphic sites were carried out with the FOXI1 resequencing data and the DnaSP software ver. 4.00 [24]. Departures from neutrality were tested by means of Tajima's D, Fu and Li's F, F*, D and D* and Fay and Wu's $\mathrm{H}$ tests. In order to obtain realistic distributions for the statistics and thus evaluate evidence for natural selection, we performed 10,000 coalescent simulations using Cosi version 1.1 [25]. As some demographic effects (such as population expansions) and positive selection have similar effects on genealogies [26] those simulations included the ad-hoc, continent-specific human demographic calibration described in Schaffner et al. [25] and that is provided with the Cosi source code. We used an infinite-sites model, with $\mathrm{S}$ fixed as the number of segregating sites found, and the length of the simulated sequence was set to the length of the sequence analyzed $(4,007 \mathrm{bp})$. The recombination rates used were those estimated for the region [27]. The critical value for each statistic was obtained from the empirical distribution of the corresponding neutral model with a significance level of 0.05 . For the whole human sample, DnaSP software ver. 4.00 [24] was used to produce coalescent neutral simulations with a constant population size.

For every population we analyzed the distribution of the minor allele frequencies (MAF) and the derived allele frequencies (DAF) of the corresponding SNPs along $2 \mathrm{Mb}$ centered on FOXI1. The proportion of SNPs with allele frequencies higher or lower than a defined threshold (MAF $<0.10$ for the MAF analysis and DAF $>0.80$ for the DAF analysis) was calculated within sliding windows of $100 \mathrm{~kb}$ in size every $20 \mathrm{~kb}$ and plotted against distance as in Moreno-Estrada et al. [28]. We constructed independent distributions for each population with additional genotype data generated elsewhere [28]. Regions enriched by windows in the top 5\% of the corresponding distribution were considered as outliers. Given their small sample size ( $<10$ individuals), the San population was not included in the MAF threshold analysis. Ancestral states for all analyzed SNPs were obtained from the chimpanzee and/or the macaque genome sequences (panTro2, Mar. 2006 assembly and rheMac2, Jan. 2006 assembly, respectively).

Unusually long range haplotypes along the $2 \mathrm{Mb}$ region centered on FOXI1 were explored by applying two complementary EHH-based approaches especially designed to detect intermediate frequency and fixed (or nearly so) selected variants. For the first approach, we applied both the Long Range Haplotype (LRH) test [29] and the iHS statistic [30], and for the second we used the XP-Rsb statistic [28]. The LRH test was carried out using the SWEEP software package (version 1.1) defining cores as the longest non-overlapping core haplotypes with at least one SNP and not more than 20 SNPs. In order to obtain additional data for a background distribution of EHH values we used phased haplotype data for eleven $2 \mathrm{Mb}$ regions studied elsewhere [28]. For each core haplotype identified, we calculated the EHH and the relative EHH (REHH) at varying distances from the core for each main continental region separately. More specifically, marker $\mathrm{H}$ (defined as the degree to which each added marker at a further distance causes the extended haplotype to decay for all core haplotypes) of 0.04 and 0.02 were selected as they are roughly equivalent to a genetic distance of 0.25 and $0.3 \mathrm{cM}$, respectively (matching the observed amount of recombination in the actual tested data). Core haplotypes were placed in $5 \%$ frequency bins and the respective $\mathrm{EHH}$ and REHH values were log-transformed for each bin in order to obtain approximately normally distributed values. The empirical significance of the LRH test was 
estimated by means of $\mathrm{p}$ and $\mathrm{q}$ values as in MorenoEstrada et al. [28]. The Integrated Haplotype Score (iHS) was estimated as previously described [30,31] using publicly available genotype data for $\sim 650,000$ genome-wide distributed SNPs in 39 populations from the HGDP-CEPH diversity panel [32]. With an approach similar to that described by Nielsen et al. [33] to detect regions with aberrant allele frequency spectra (test 1 ), we applied a composite likelihood test to detect regions with aberrant "iHS spectra". We first divided |iHS| in bins of size 0.1 , and then estimated the probability that a SNP had an $|\mathrm{iHS}|$ value in a particular bin, both for each SNP in the whole genome dataset (which produced an empirical background distribution) as well as for each sliding window of 31 SNPs over the whole genome. Two composite likelihoods were estimated for each window, one by multiplying the probabilities obtained from the window itself, and the other by multiplying the probabilities obtained from the whole genome. A loglikelihood ratio was then formed comparing both likelihoods, where extreme values indicate unusual distributions of iHS in the respective window compared to the rest of the genome. For each SNP this log-likelihood ratio for the window centered on the SNP is referred to as windowed iHS in Results. Finally, in order to allow for multiple population comparisons of $\mathrm{EHH}$, we computed XP-Rsb for every SNP site and population versus all other HGDP-CEPH populations as described in Moreno-Estrada et al. [28]. In order to assess significance, we obtained a $\mathrm{p}$-value for each population and SNP site along the FOXI1 region by ranking its XP-Rsb value with respect to the genome-wide distribution generated with the $\sim 650,000$ SNPs from Li et al. [32]. We then log transformed the p-values and plotted them against position, searching for clusters of significant values inside or around the FOXI1 gene.

\section{Results}

\section{FOXI1 divergence patterns}

The recent increase of available newly sequenced genomes led us to re-evaluate the initial evidence of nonneutral evolution of FOXI1 in the human lineage obtained with three-species sequence alignments [5]. When considering a phylogeny of five mammals, the improved branch-site test 2 of positive selection [14] rejected the hypothesis of positive selection at a subset of sites in the human branch $(\mathrm{p}=0.7039)$. We also investigated the patterns of FOXI1 protein evolution from several multiple sequence alignments, including additional non-human primates besides chimpanzee. We used two maximum likelihood methods (see Materials and Methods) to specifically test for: (i) a $\mathrm{d}_{\mathrm{N}} / \mathrm{d}_{\mathrm{S}}$ ratio on the human branch significantly different from 1 , and (ii) codon sites with a $d_{N} / d_{S}$ ratio significantly different from 1 in the human lineage. Overall FOXI1 did not present significantly accelerated amino acid substitution rates or particular codon sites undergoing positive selection in the human branch in any of the comparisons (Additional file 5, Table S3).

\section{Patterns of FOXI1 sequence variation}

Polymorphic variation in the FOXI1 gene was investigated by sequencing $4,007 \mathrm{bp}$, encompassing its two exons, most of its untranslated regions, and its corresponding intronic region, in 20 Yorubas, 20 Asians and 20 Europeans. We found 22 sequence variations: 21 substitutions and one deletion/insertion polymorphism (Table 1). Among them, six were singletons: one in Yorubas, three in Europeans and two in Asians. One of the Asian-specific singletons was the only non-synonymous substitution observed (rs3828625), an Asn to Ser replacement at amino acid position 362 for isoform a (or amino acid position 267 for isoform b). The remaining three exonic polymorphisms were synonymous changes. Twenty of the 22 sequence variations detected here had been previously reported in dbSNP build 131. Excluding singleton variants, we identified a total of 15 haplotypes (Table 2). Half of the analysed chromosomes belonged to either $\mathrm{Ht}-01$ or $\mathrm{Ht}-04$, found in all three populations studied. We found three specific haplotypes for Africans, three for Europeans, but none specific for Asians. In order to visualize the phylogenetic relationships among the identified haplotypes we constructed a median-joining network; ht-08 carried ancestral alleles at each SNP (Additional File 6, Figure S3). The reticulated pattern observed in the network points to the action of recurrent mutation or recombination. However, given that more than one nucleotide position is often involved, the latter seems more likely as a mechanism for producing new sequence variation.

Summary statistics of genetic diversity and neutrality tests for FOXI1 are reported in Table 3. These levels of diversity are in agreement with estimates from other genomic regions sequenced in a similar set of samples (SeattleSNPs database, http://pga.gs.washington.edu/). Notably, in the Yoruban population the number of segregating sites, and different haplotypes as well as values of nucleotide diversity were similar to the European population sample. However, in Africans haplotype diversity was lower. In order to investigate the possible genetic footprint of selection we performed several neutrality tests on each of these three populations and on the whole human sample (Table 3). Significance was estimated by means of coalescent simulations under each inferred past demography [25] or in the case of the whole human sample by considering constant population size. None of the neutrality statistics displayed significant departure from neutrality $(\mathrm{P}>0.05)$. 
Table 1 Summary of FOXI1 polymorphisms

\begin{tabular}{|c|c|c|c|c|c|c|c|c|}
\hline \multirow[b]{2}{*}{$\mathbf{N}^{\circ}$} & \multirow[b]{2}{*}{ Position $^{a}$} & \multirow[b]{2}{*}{ dbSNP code } & \multirow[b]{2}{*}{ Type of change ${ }^{b}$} & \multirow[b]{2}{*}{ Nucleotide change $^{c}$} & \multicolumn{3}{|c|}{ Derived Allele Frequency } & \multirow[b]{2}{*}{$\mathrm{F}_{\mathrm{ST}}{ }^{\mathrm{d}}$} \\
\hline & & & & & Yoruban $(2 \mathrm{~N}=40)$ & European $(2 \mathrm{~N}=40)$ & Asian $(2 \mathrm{~N}=40)$ & \\
\hline 1 & 169465818 & rs2277944 & CS & $\mathrm{G} / \mathrm{A}$ & 0.58 & 0.20 & 0.35 & 0.123 \\
\hline 2 & 169466184 & rs2277945 & । & $\mathrm{T} / \mathrm{C}$ & 0.23 & 0.28 & 0.43 & 0.026 \\
\hline 3 & 169466367 & rs2112669 & । & $C / G$ & 0.55 & 0.23 & 0.33 & 0.092 \\
\hline 4 & 169466686 & rs11951903 & । & $\mathrm{T} / \mathrm{C}$ & 0.05 & 0.00 & 0.25 & 0.162 \\
\hline 5 & 169466751 & rs4315934 & । & $A / G$ & 0.88 & 0.55 & 0.78 & 0.113 \\
\hline 6 & 169466837 & rs4315935 & । & $A / G$ & 0.88 & 0.55 & 0.78 & 0.113 \\
\hline 7 & 169467111 & rs2879269 & । & $A / G$ & 0.03 & 0.10 & 0.00 & 0.042 \\
\hline 8 & 169467123 & rs7380481 & । & $\mathrm{T} / \mathrm{C}$ & 0.23 & 0.28 & 0.43 & 0.026 \\
\hline 9 & 169467572 & rs77136537 & 1 & $\mathrm{G} / \mathrm{A}$ & 0.00 & 0.03 & 0.00 & 0.000 \\
\hline 10 & 169468070 & rs55685928 & $\mathrm{CS}$ & $G / A$ & 0.05 & 0.00 & 0.00 & 0.026 \\
\hline 11 & 169468100 & rs10063424 & CS & $\mathrm{C} / \mathrm{T}$ & 0.10 & 0.10 & 0.00 & 0.028 \\
\hline 12 & 169468141 & rs3828625 & CNS & $\mathrm{A} / \mathrm{G}$ & 0.00 & 0.00 & 0.03 & 0.000 \\
\hline 13 & 169468312 & rs6873124 & UTR & $C / A$ & 0.53 & 0.20 & 0.33 & 0.091 \\
\hline 14 & 169468336 & rs55762796 & UTR & $\mathrm{A} / \mathrm{T}$ & 0.05 & 0.08 & 0.03 & -0.012 \\
\hline 15 & 169468524 & rs72828668 & UTR & $\mathrm{G} / \mathrm{C}$ & 0.00 & 0.00 & 0.03 & 0.000 \\
\hline 16 & 169468633 & rs6555887 & UTR & $A / G$ & 0.15 & 0.10 & 0.00 & 0.051 \\
\hline 17 & 169468728 & rs6555888 & UTR & $\mathrm{A} / \mathrm{G}$ & 0.83 & 0.70 & 0.95 & 0.082 \\
\hline 18 & 169468749 & rs77823283 & UTR & $A / G$ & 0.00 & 0.03 & 0.00 & 0.000 \\
\hline 19 & 169468769 & & UTR & $\mathrm{T} / \mathrm{A}$ & 0.00 & 0.03 & 0.00 & 0.000 \\
\hline 20 & 169469034 & & UTR & $\mathrm{T} / \mathrm{A}$ & 0.03 & 0.00 & 0.00 & 0.000 \\
\hline 21 & 169469117 & rs45466695 & UTR & $\mathrm{C} / \mathrm{T}$ & 0.000 & 0.08 & 0.00 & 0.051 \\
\hline 22 & 169469123 & rs3839285 & UTR & $-/ T$ & 0.53 & 0.20 & 0.33 & 0.091 \\
\hline
\end{tabular}

Positions are based on NCBI build 36.3

${ }^{\mathrm{b}} \mathrm{CNS}$ stands for coding non-synonymous; CS, synonymous; I, intronic; and UTR, untranslated region

${ }^{\text {CT}}$ The allele named first corresponds to the ancestral state for each SNP

${ }^{\mathrm{d} O \text { Overall }} \mathrm{F}_{\mathrm{ST}}$ among Yorubans, Europeans and Asians

Table 2 Summary of human FOXI1 haplotypes

\begin{tabular}{|c|c|c|c|c|c|c|c|c|c|c|c|c|c|c|c|c|c|c|c|c|}
\hline Anc & $\begin{array}{l}1 \\
\mathrm{G}\end{array}$ & $\begin{array}{l}2 \\
T\end{array}$ & $\begin{array}{l}3 \\
C\end{array}$ & $\begin{array}{l}4 \\
T\end{array}$ & $\begin{array}{l}5 \\
\text { A }\end{array}$ & $\begin{array}{l}6 \\
A\end{array}$ & $\begin{array}{l}7 \\
\text { A }\end{array}$ & 8 & $\begin{array}{c}10 \\
\text { G }\end{array}$ & $\begin{array}{l}11 \\
\mathrm{C}\end{array}$ & $\begin{array}{c}13 \\
\text { C }\end{array}$ & $\begin{array}{l}14 \\
\mathrm{~A}\end{array}$ & $\begin{array}{c}16 \\
\text { A }\end{array}$ & $\begin{array}{l}17 \\
\mathrm{~A}\end{array}$ & $\begin{array}{c}21 \\
\text { C }\end{array}$ & $\begin{array}{c}22 \\
-\end{array}$ & Europe & Africa & Asia & Total \\
\hline $\mathrm{Ht}-01$ & A & & G & . & G & G & . & . & . & . & A & . & . & G & . & $\mathrm{T}$ & 8 & 19 & 3 & 30 \\
\hline $\mathrm{Ht}-02$ & A & & G & C & G & G & . & . & . & . & A & . & & G & . & $\mathrm{T}$ & 0 & 2 & 10 & 12 \\
\hline $\mathrm{Ht}-03$ & A & C & . & . & G & G & . & C & . & & . & . & & G & . & & 0 & 1 & 1 & 2 \\
\hline $\mathrm{Ht}-04$ & . & C & . & . & G & G & . & C & . & & . & . & & G & . & & 10 & 8 & 16 & 34 \\
\hline $\mathrm{Ht}-05$ & & C & & & G & G & & C & & . & & & . & & & . & 1 & 0 & 0 & 1 \\
\hline $\mathrm{Ht}-06$ & & . & & & G & G & & & . & . & & $\mathrm{T}$ & . & G & & . & 3 & 2 & 1 & 6 \\
\hline $\mathrm{Ht}-07$ & . & . & . & . & . & . & . & . & . & & . & . & & G & . & & 7 & 1 & 7 & 15 \\
\hline $\mathrm{Ht}-08$ & & . & . & . & . & & & & . & . & & . & . & & & . & 6 & 1 & 2 & 9 \\
\hline $\mathrm{Ht}-09$ & . & . & G & . & . & . & . & . & . & . & . & . & & . & . & & 1 & 0 & 0 & 1 \\
\hline $\mathrm{Ht}-10$ & . & . & . & . & G & G & . & . & . & . & . & . & G & . & . & & 0 & 2 & 0 & 2 \\
\hline $\mathrm{Ht}-11$ & . & . & . & . & . & . & . & . & . & $\mathrm{T}$ & . & . & G & . & . & & 0 & 1 & 0 & 1 \\
\hline $\mathrm{Ht}-12$ & . & . & . & . & . & . & . & . & A & $\mathrm{T}$ & . & . & G & . & . & & 0 & 1 & 0 & 1 \\
\hline $\mathrm{Ht}-13$ & . & . & . & . & . & . & G & . & . & $\mathrm{T}$ & . & . & G & . & . & & 1 & 1 & 0 & 2 \\
\hline $\mathrm{Ht}-14$ & . & . & . & . & . & . & G & . & . & $\mathrm{T}$ & . & . & G & . & $\mathrm{T}$ & & 3 & 0 & 0 & 3 \\
\hline $\mathrm{Ht}-15$ & A & & G & & G & G & & & A & $\mathrm{T}$ & & & G & & & . & 0 & 1 & 0 & 1 \\
\hline
\end{tabular}

Note: Each polymorphic variant is displayed below the corresponding ancestral position. Ancestral-like alleles are indicated with dots. 
Table 3 Population sequence variation and neutrality test statistics for FOXI1

\begin{tabular}{|c|c|c|c|c|c|c|c|c|c|c|c|c|}
\hline Population & $2 \mathrm{~N}^{\mathrm{a}}$ & $S^{b}$ & $\pi^{c}$ & $K^{d}$ & $\mathrm{H}^{\mathrm{e}}$ & $\begin{array}{c}\text { Tajima's } \\
\text { D }\end{array}$ & $\begin{array}{c}\text { Fu and Li's } \\
D^{*}\end{array}$ & $\begin{array}{c}\text { Fu and Li's } \\
\text { D }\end{array}$ & $\begin{array}{c}\text { Fu and Li's } \\
\mathrm{F}^{*}\end{array}$ & $\begin{array}{c}\text { Fu and Li's } \\
\text { F }\end{array}$ & $\begin{array}{l}\text { Fu's } \\
\text { Fs }\end{array}$ & $\begin{array}{c}\text { Fay and Wu's } \\
\text { H }\end{array}$ \\
\hline European & 40 & 17 & $\begin{array}{c}0.0012 \pm \\
0.0001\end{array}$ & 12 & $\begin{array}{l}0.874 \pm \\
0.026\end{array}$ & 0.485 & 0.431 & 0.433 & 0.530 & 0.543 & -0.421 & 1.600 \\
\hline Asian & 40 & 13 & $\begin{array}{c}0.0010 \pm \\
0.0001\end{array}$ & 8 & $\begin{array}{l}0.768 \pm \\
0.045\end{array}$ & 1.134 & 0.065 & 0.030 & 0.486 & 0.485 & 1.969 & -1.923 \\
\hline Yoruban & 40 & 16 & $\begin{array}{c}0.0011 \pm \\
0.0001\end{array}$ & 12 & $\begin{array}{l}0.741 \pm \\
0.063\end{array}$ & 0.487 & 0.763 & 0.799 & 0.793 & 0.831 & -0.664 & -2.928 \\
\hline All & 120 & 22 & $\begin{array}{c}0.0012 \pm \\
0.0001\end{array}$ & 19 & $\begin{array}{c}0.837 \pm \\
0.019\end{array}$ & 0.360 & -0.808 & -0.863 & -0.430 & -0.458 & -1.417 & -0.410 \\
\hline
\end{tabular}

Note: At $P=0.05$, none of the neutrality statistics were significant

${ }^{a}$ Number of chromosomes

${ }^{\mathrm{b}}$ Number of segregating sites

${ }^{\mathrm{C}}$ Nucleotide diversity per base pairs

${ }^{\mathrm{d}}$ Total number of haplotypes

${ }^{\mathrm{H}}$ Haplotype diversity

\section{Patterns of SNP variation in FOXI1}

In order to widen both the genomic context and the range of human populations analyzed, we also explored the pattern of SNP variation along a $2 \mathrm{Mb}$ region centered on FOXI1 in 39 worldwide human populations covering more human genetic diversity than the three samples we sequenced. In particular, we looked for an excess of low frequency variants, the presence of high frequency derived alleles, and unusually long range haplotypes. These possible signatures of natural selection persist in the human genome at varying time scales, and therefore give information about adaptative events occurring at different intervals during our evolutionary history [34]. For each population and within multiple $100 \mathrm{~kb}$ sliding windows along the FOXI1-centered $2 \mathrm{Mb}$ region we separately plotted: (i) the proportion of SNPs with MAF $<0.10$ (Additional File 7, Figure S4) and (ii) the proportion of SNPs with DAF $>0.80$ (Additional File 8 , Figure S5). In both analyses, no particular pattern emerged around or surrounding the FOXI1 gene in any of the populations studied. However, when searching for signatures of unusually long haplotypes, we found strong signals from all the three statistics we applied. First, according to the LRH test [29], up to five different core haplotypes close to FOXI1 clearly stood out with relatively high frequencies $(>0.50)$ and significant $\mathrm{REHH}$ values in the Yoruba population (see Table 4 and Materials and Methods). A picture of the unusual EHH breakdown around FOXI1 in Yorubas is presented in Figure 1. Similarly, in Sub-Saharan Africans (SSAFR) from the HGDP-CEPH diversity panel, the complete FOXI1 gene region was clearly enriched for outliers of the iHS statistic [30], reaching significant values within the top $1 \%$ of the genome-wide distribution (iHS >3) (see Figure 2). The strongest signals were located inside FOXI1 and extended several kb downstream in agreement with the location of the most significant core haplotypes observed with the LRH test described above.

Table 4 Core haplotypes with significant REHH values in Africans involving FOXI1

\begin{tabular}{|c|c|c|c|c|c|c|c|c|}
\hline $\mathrm{H}^{\mathrm{a}}$ & REHH & Frequency & Distance $(b p)^{b}$ & Distance $(\mathrm{cM})^{\mathrm{c}}$ & Core haplotype & Genes in core region ${ }^{i}$ & $P$ value & $q$ value $^{\mathrm{j}}$ \\
\hline 0.02 & 19.58 & 0.528 & 80443 & 0.056 & $\mathrm{ACCC}^{\mathrm{d}}$ & DOCK2, FOXI1 & $0.8 \times 10^{-5}$ & 0.0038 \\
\hline 0.04 & 16.93 & 0.528 & 61086 & 0.026 & $\mathrm{ACCC}^{\mathrm{d}}$ & DOCK2, FOXI1 & $0.3 \times 10^{-5}$ & 0.0016 \\
\hline 0.04 & 12.08 & 0.590 & -64129 & -0.058 & $A G C^{e}$ & FOXI1 & $0.6 \times 10^{-4}$ & 0.0213 \\
\hline 0.04 & 8.32 & 0.675 & -41241 & -0.051 & $A G^{f}$ & DOCK2, FOXI1 & $0.8 \times 10^{-4}$ & 0.0247 \\
\hline 0.04 & 10.42 & 0.528 & 55531 & 0.028 & $A^{g}$ & DOCK2, FOXI1 & $0.8 \times 10^{-4}$ & 0.0191 \\
\hline 0.04 & 10.65 & 0.561 & -54967 & -0.053 & $\mathrm{CTG}^{\mathrm{h}}$ & DOCK2, FOXI1 & $1.2 \times 10^{-4}$ & 0.0215 \\
\hline
\end{tabular}

${ }^{a}$ Degree to which each added marker at a further distance causes the extended haplotypes to decay for all core haplotypes

${ }^{b}$ Physical distance (bp) from the core at which the signal has been captured. (-) indicates downstream direction. Otherwise upstream

${ }^{\mathrm{C}} \mathrm{Genetic}$ distance (cM) from the core at which the signal has been captured. (-) indicates downstream direction. Otherwise upstream

drs7736379, rs6872596, rs4449553, rs17562083

ers7729440, rs2879278, rs11134616

frs4867919, rs11134612

${ }^{g} \mathrm{rs} 1501644$

hrs7709558, rs12515896, rs6861611

'Genes within $\pm 100 \mathrm{~kb}$ around the core are considered

${ }^{j}$ Expected proportion of false positives among all significant $p$ values 


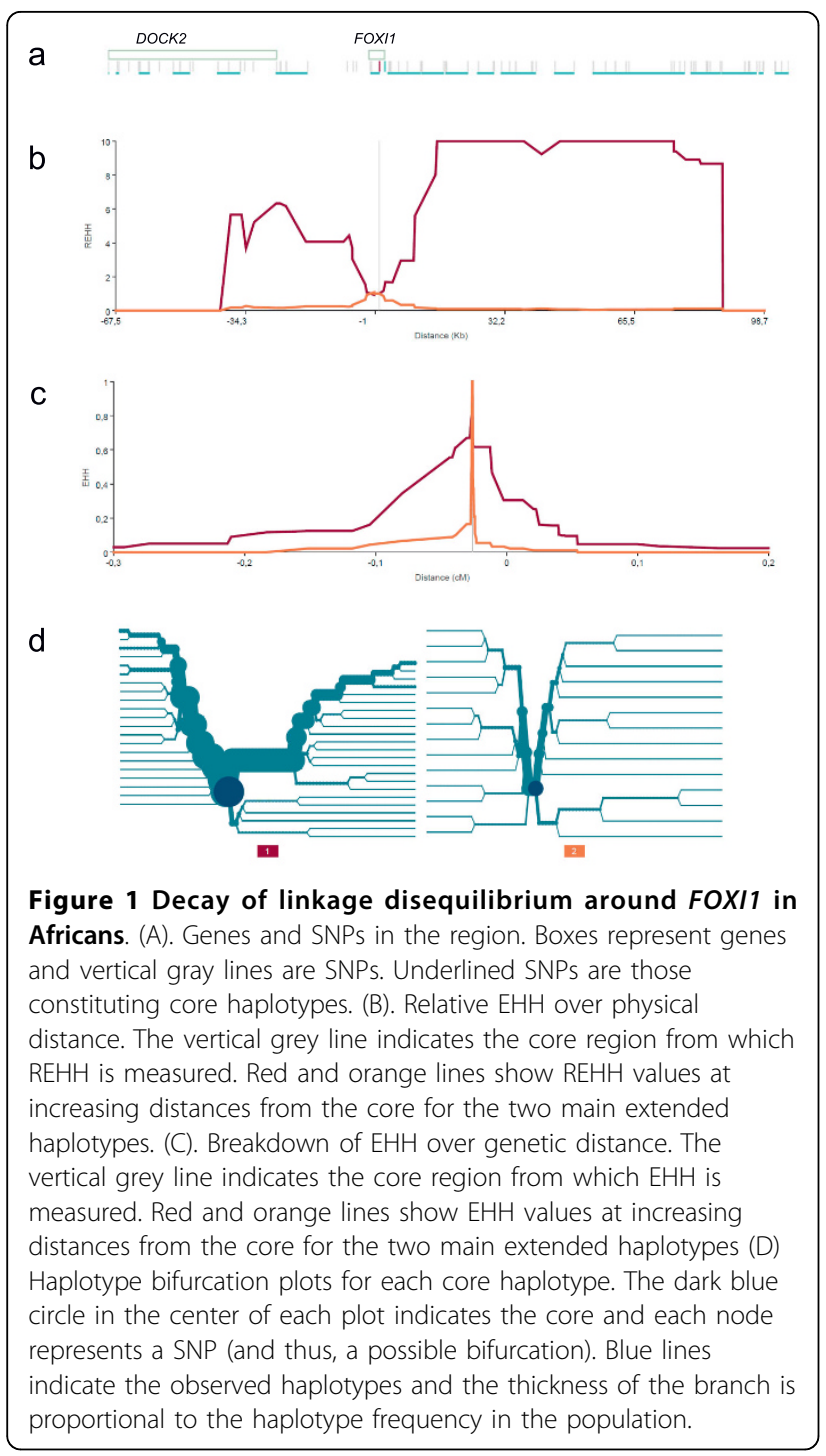

When ranking all genes in the genome by their maximum windowed iHS within the gene region (defined as the longest transcript $\pm 2 \mathrm{~kb}$ ), FOXI1 was ranked number 339 out of 17,637 (p-value $=0.019)$ in the SSAFR continental sample. Finally, we used the XP-Rsb statistic [28] to compare the integrated $\mathrm{EHH}$ at each particular SNP site in a population with that averaged across multiple populations (as opposed to comparing the EHH of alleles within the same population like in the LRH test and the iHS statistic). Interestingly, a cluster of significant XP-Rsb values was detected in the FOXI1 region for the Yoruba $(\mathrm{p}<0.01)$ and Mandenka $(\mathrm{p}<0.05)$ African populations. Figure 3 shows genome-wide significance for XP-Rsb at every SNP site and population plotted against distance along the $2 \mathrm{Mb}$ region centered in FOXI1. The cluster of significant values involving FOXI1 was exclusively observed in Africans and also correlates with the location of the LRH and iHS signals previously described. Other significant values can be seen in Figure 3, for example, at $168.5 \mathrm{Mb}$ for the French population, and around $170 \mathrm{Mb}$ for $\mathrm{N}$. Italy. These signals lie well beyond FOXI1 and, given the pattern of linkage disequilibrium in the region, are most certainly unrelated to this gene.

We next explored what may have been the functional variation underlying the recent selection signal detected in Africa by these two EHH-based approaches. To that effect, using HapMap data [35] we studied the linkage disequilibrium (LD) pattern around FOXI1 in the Yoruban population and found a LD block containing FOXI1 in a region of $\sim 70 \mathrm{~kb}$ between two hotspots of recombination. As shown in Figure S6 (Additional File 9), a similar LD block structure was detected in the Yoruban population of the HGDP-CEPH panel. Then we screened all known SNP variation for a putative functional effect using PupaSuite [36,37] in a $\sim 140 \mathrm{~kb}$ segment containing the previously identified LD block and all the significant core haplotypes found in the FOXI1 gene region (see Additional File 9, Figure S6). Out of 646 SNPs we detected 19 as functionally relevant polymorphisms (Additional File 10, Table S4). Since we have found that the footprint of natural selection seems to be evident in Africans, we can narrow down that candidate list by rejecting the SNPs with small frequency differences between Africans and non-Africans. After removing the SNPs with an absolute difference in MAF between Africans and Europeans smaller than 0.2, only six SNPs remained. Five of them affect exonic splicing enhancers either for the DOCK2 gene (rs6555882, rs1045176 and rs9307) or the FOXI1 gene (rs2277944 and rs6873124), while rs7704953 creates a new transcription factor binding site for FOXI1. Except for rs6555882, which maps further away, these SNPs are all within the $70 \mathrm{~kb}$ block described above. Therefore, they (or unknown functional variants in linkage disequilibrium with them) are the best candidates to represent the functional variation that caused this signature of selection.

Finally, in order to explore a possible relation between climate and FOXI1 variation, we tested for correlation between allele frequencies and absolute latitude for the SNPs in the significant core haplotypes in the LRH test, SNPs with significant values for the iHS and XP-Rsb statistics, and rs2277944 (worldwide allele frequency distributions were not available for the other suggested candidate functional SNPs). Out of 27 SNPs, correlation with absolute latitude was nominally significant ( $\mathrm{p}<$ 0.05 ) for 13 of them. All significant core haplotypes contained at least one SNP with significant correlation with absolute latitude $(\mathrm{p}<0.05)$, and rs2277944 was also significantly correlated with latitude $(\mathrm{p}<0.01)$. The most 


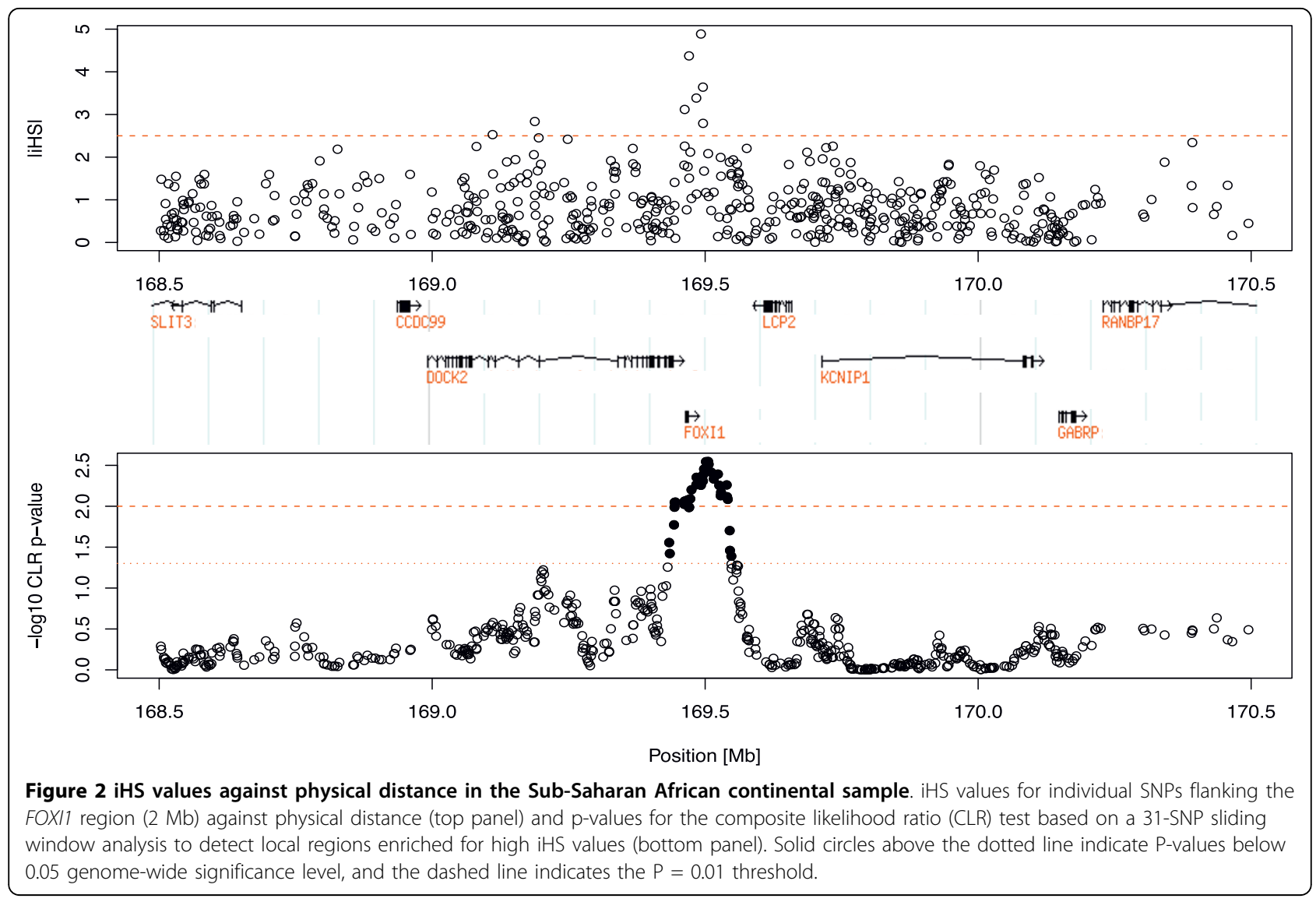

significant correlation (Pearson's $\mathrm{r}=-0.536, \mathrm{p}<0.01$ after Bonferroni correction) was for allele $\mathrm{A}$ at rs7736379 in core ACCC (see Table 4), implying that its frequency decreases towards higher latitudes (Figure 4). Since the most significant event to shape human genetic diversity was the Out of Africa migration, we recalculated partial correlation coefficients with absolute latitude by controlling for the geographical distance to East Africa (Nairobi). All the significant correlations mentioned above remained significant, implying that the dispersion out of Africa is not a meaningful confounding factor for this result. However, when put in a genomic context, these correlations are not exceptional: out of 585,379 SNPs with minor allele frequency $>0.05$ genotyped in the same individuals by $\mathrm{Li}$ et al. [32], 8.6\% had more extreme correlations with absolute latitude, and $14 \%$ after correcting for distance to East Africa.

\section{Discussion}

Genes related to fertility and/or sensory perception may have been driven by accelerated evolution in humans, but, contrary to Clark et al. [5], we did not find in the human branch such acceleration of the FOXI1 gene, which is involved in the function of the ear, testis, and kidney [8]. It is recognised that the accuracy and power of likelihood ratio tests (LRTs) for detecting positive selection improve with the number of species used in such approaches [38]. Consequently, we used the Human PAML browser [17] in order to perform both branch and branch-site tests of positive selection in multiple species comparisons containing several mammals and additional primates to human and chimpanzee. None of the tests performed indicated positive selection for the FOXI1 coding sequence in the lineage leading to humans. However, $\omega$ is $\sim 1$ in many branches of the FOXI1 phylogeny (see Additional File 3, Figure S1), which suggests that the FOXI1 amino acid replacement rate is equally fast in all branches and that comparative LRTs such as the ones performed here may lack power to detect positive selection in any particular lineage. In any case, we did not find evidence of a specific evolution pattern for FOXI1 in the lineage leading to humans.

Variation in the FOXI1 gene sequence was compatible with neutral evolution in samples of Africans, East Asians and Europeans. However, patterns of extended linkage disequilibrium in the FOXI1 gene region suggested recent positive selection in some of the African samples of the HGDP-CEPH diversity panel [10]. Half of the SNPs with significant values for the iHS or XPRsb statistics and of those contained in significant cores 


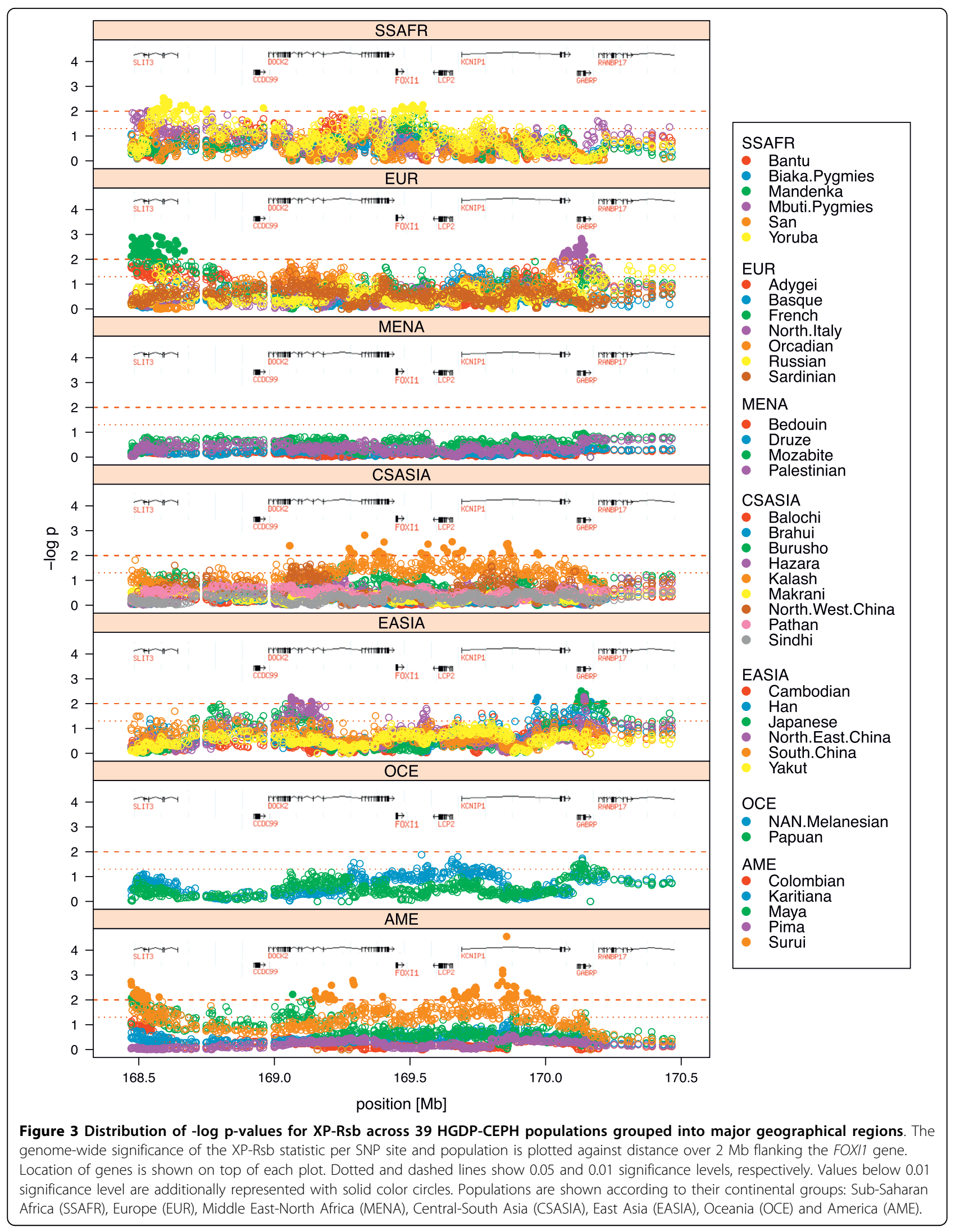




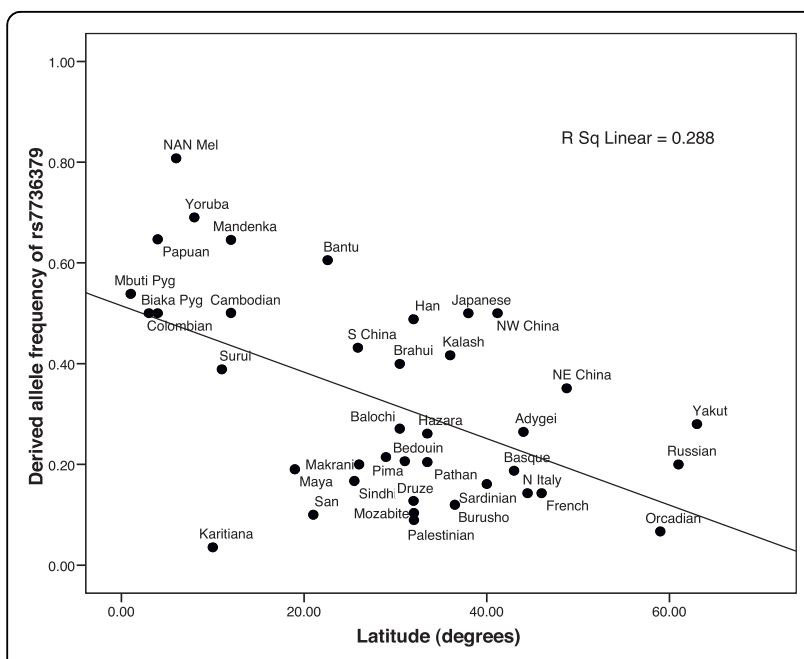

Figure 4 Allele frequencies at rs7736379 as a function of absolute latitude in 39 HGDP-CEPH populations. The line represents linear regression.

haplotypes in the LRH test correlated with latitude, suggesting a possible role for climate in the adaptation mediated by FOXI1. A higher correlation with latitude may not have been reached due to the apparent recentness of the selective event and its restriction to SubSaharan African populations. An alternative explanation (but less likely, given the distance to most of the significant signals of selection found) is geographically localized selection at the DOCK2 gene, which encodes for a cytoplasmatic protein required for lymphocyte chemotaxis in response to chemokines [39]. It is suggested that patterns of linkage disequilibrium pinpoint recent selective events, while the accumulation of variation by mutation requires some time before sequence-based neutrality statistics become significant [40]. The finding of no significant deviation from neutral evolution with statistics such as Tajima's D, or Fay and Wu's $\mathrm{H}$ has been previously recognized to be consistent with the low power of these traditional tests to detect recent selective sweeps as for example in the case of the G6PD locus [29]. Moreover, tests based on the allele frequency spectrum have low power to detect incomplete sweeps, especially after a selective event in standing genetic variation [40]. Interestingly, while nucleotide and haplotype diversities tend to be higher in Africans than in other populations, this was not the case for FOXI1, which could be taken as further evidence of positive selection in Africans. No non-synonymous coding SNPs were found in the core haplotypes or their linked variation. Therefore, taken as a whole, our results suggest that recent selection may have acted on the expression rather than on the amino acid sequence of FOXI1. Based on two extensive collections of microarray data from a large variety of available human tissue samples,
Genevestigator [41] and GeneSapiens [42], FOXI1 has the highest mRNA expression levels in kidney (but inner ear data was not available). Fittingly, among the diverse functions of this transcription factor, its role in the kidney as a master regulator of vacuolar $\mathrm{H}+$-ATPase proton pump subunits [8] might be an intriguing candidate function for climate adaptation through water-electrolyte homeostasis and prevention of dehydration. If that were the case, FOXI1 would join the ranks of the growing number of known genes such as $F A B P 2$, RAPTOR and SLC24A5 [43] that allowed humans to adapt to the diversity of climates we encountered during our past expansion.

\section{Conclusions}

We have found no genetic evidence of accelerated protein evolution for the FOXI1 gene in the human lineage; however, within humans we have discovered signatures of a recent episode of positive selection in African populations. Our evolutionary approach identifies directions for future functional analyses of how genetic variation in the FOXI1 gene region can result in phenotypic differences among human populations, which might be related to water-electrolyte homeostasis.

\section{Additional material}

Additional file 1: Table S1: Coriell repository numbers.

Additional file 2: Table S2: Amplification and sequencing primers.

Additional file 3: Figure S1: Phylogeny of the five mammalian species used in PAML analysis. Each branch is labeled with the corresponding estimate of $\omega$ calculated under a free branch model [44] with the codeml program within PAML using standard parameters [28] Inferred synonymous and non-synonymous substitutions are indicated within brackets.

Additional file 4: Figure S2: Alignment of the five FOXI1 mammalian sequences used in PAML analysis.

Additional file 5: Table S3: Significance of the likelihood ratio tests of positive selection performed on the human lineage for the FOXI1 gene.

Additional file 6: Figure S3: Median Joining Network of human FOXI1 haplotypes. Nodes in the median joining network are proportional to frequencies and branch lengths to the number of polymorphic base substitutions.

Additional file 7: Figure S4: Distribution of low-frequency minor alleles. The proportion of SNPs with a minor allele frequency (MAF) of less than 0.10 within $100 \mathrm{~kb}$ sliding windows is plotted for each population. Solid dots represent values above the 95th percentile for each population, whereas open dots are values below the 95th percentile.

Additional file 8: Figure S5: Distribution of high-frequency derived alleles. The proportion of SNPs with a derived allele frequency (DAF) greater than 0.80 within $100 \mathrm{~kb}$ sliding windows is plotted for each population. Solid dots represent values above the 95th percentile for each population, whereas open dots are values below the 95th percentile.

Additional file 9: Figure S6: Pattern of linkage disequilibrium around FOXI1 in the Yoruba population from the CEPH-HGDP diversity panel. Linkage disequilibrium from position 169,401,507 to 
169,544,856 on chromosome 5, NCBI build 36.3. Green boxes represent significant core haplotypes from the LRH test and are labelled with letters d-h as in Table 4.

Additional file 10: Table S4: Functional characterization and allele frequencies for functionally relevant SNPs within $\sim 140 \mathrm{~kb}$ containing the FOXI1 gene.

\section{Acknowledgements}

We specially thank M Vallès for her technical support as well as K Tang, L Montanucci and A Navarro for statistical advice. This research was funded by grant BFU2005-00243 awarded by Dirección General de Investigación, Ministerio de Educación y Ciencia (Spain), by grant BFU2008-01046/BMC awarded by Subdirección General de Proyectos de Investigación, Ministerio de Ciencia e Innovación (Spain), and by the Direcció General de Recerca, Generalitat de Catalunya (2009SGR1101). AME was supported by a CONACYT fellowship from the Mexican government (grant 179339), MS by a PhD fellowship from the Programa de becas FPU del Ministerio de Educación y Ciencia, Spain (AP2005-3982) and JE by a Volkswagenstiftung scholarship (I/82 750). SNP genotyping services were provided by the Spanish "Centro Nacional de Genotipado" (http://www.cegen.org).

\section{Author details}

Institut de Biologia Evolutiva (UPF-CSIC), Departament de Ciències Experimentals i de la Salut, Universitat Pompeu Fabra, Parc de Recerca Biomèdica de Barcelona, C/Dr. Aiguader 88, 08003 Barcelona, Spain. ${ }^{2}$ Centro de Investigación Biomédica en Red de Epidemiología y Salud Pública (CIBERESP), Barcelona, Catalonia, Spain. ${ }^{3}$ Department of Genetics, Stanford University School of Medicine, USA.

\section{Authors' contributions}

AME participated in the design of the study, carried out the analysis of the SNP and resequencing data and helped to draft the manuscript. EAP performed the resequencing analysis. MS, JE and ARS participated in the statistical analysis. FC helped in the statistical analysis and in drafting the manuscript. EB participated in the design, analysis and coordination of the study and drafted the manuscript. All authors read and approved the final manuscript.

Received: 15 April 2010 Accepted: 1 September 2010 Published: 1 September 2010

\section{References}

1. Katoh M, Katoh M: Human FOX gene family (Review). Int J Oncol 2004, 25(5):1495-1500

2. Yang $\mathrm{T}$, Vidarsson $H$, Rodrigo-Blomqvist $\mathrm{S}$, Rosengren SS, Enerback $\mathrm{S}$, Smith RJ: Transcriptional control of SLC26A4 is involved in Pendred syndrome and nonsyndromic enlargement of vestibular aqueduct (DFNB4). Am J Hum Genet 2007, 80(6):1055-1063.

3. Blomqvist $S R$, Vidarsson $H$, Fitzgerald $S$, Johansson BR, Ollerstam A, Brown $R$, Persson AE, Bergstrom GG, Enerback S: Distal renal tubular acidosis in mice that lack the forkhead transcription factor Foxi1. J Clin Invest 2004, 113(11):1560-1570.

4. Blomqvist SR, Vidarsson $\mathrm{H}$, Soder $\mathrm{O}$, Enerback $\mathrm{S}$ : Epididymal expression of the forkhead transcription factor Foxi1 is required for male fertility. Embo J 2006, 25(17):4131-4141.

5. Clark AG, Glanowski S, Nielsen R, Thomas PD, Kejariwal A, Todd MA, Tanenbaum DM, Civello D, Lu F, Murphy B, et al: Inferring nonneutral evolution from human-chimp-mouse orthologous gene trios. Science 2003, 302(5652):1960-1963.

6. Arbiza L, Dopazo J, Dopazo H: Positive selection, relaxation, and acceleration in the evolution of the human and chimp genome. PLOS Comput Biol 2006, 2(4):e38.

7. Nielsen R, Bustamante C, Clark AG, Glanowski S, Sackton TB, Hubisz MJ, Fledel-Alon A, Tanenbaum DM, Civello D, White TJ, et al: A scan for positively selected genes in the genomes of humans and chimpanzees. PLOS Biol 2005, 3(6):e170.

8. Wilson TW, Grim CE: Biohistory of slavery and blood pressure differences in blacks today. A hypothesis. Hypertension 1991, 17(1 Suppl):1122-128.
9. Thompson EE, Kuttab-Boulos H, Witonsky D, Yang L, Roe BA, Di Rienzo A: CYP3A variation and the evolution of salt-sensitivity variants. Am J Hum Genet 2004, 75(6):1059-1069.

10. Cann HM, de Toma C, Cazes L, Legrand MF, Morel V, Piouffre L, Bodmer J, Bodmer WF, Bonne-Tamir B, Cambon-Thomsen A, et al: A human genome diversity cell line panel. Science 2002, 296(5566):261-262.

11. Rosenberg NA: Standardized subsets of the HGDP-CEPH Human Genome Diversity Cell Line Panel, accounting for atypical and duplicated samples and pairs of close relatives. Ann Hum Genet 2006, 70(Pt 6):841-847.

12. Gardner M, Gonzalez-Neira A, Lao O, Calafell F, Bertranpetit J, Comas D: Extreme population differences across Neuregulin 1 gene, with implications for association studies. Mol Psychiatry 2006, 11(1):66-75.

13. Moreno-Estrada A, Casals F, Ramirez-Soriano A, Oliva B, Calafell F, Bertranpetit J, Bosch E: Signatures of selection in the human olfactory receptor OR5I1 gene. Mol Biol Evol 2008, 25(1):144-154.

14. Zhang J, Nielsen R, Yang Z: Evaluation of an improved branch-site likelihood method for detecting positive selection at the molecular level. Mol Biol Evol 2005, 22(12):2472-2479.

15. Thompson JD, Higgins DG, Gibson TJ: CLUSTAL W: improving the sensitivity of progressive multiple sequence alignment through sequence weighting, position-specific gap penalties and weight matrix choice. Nucleic Acids Res 1994, 22(22):4673-4680

16. Yang Z: PAML: a program package for phylogenetic analysis by maximum likelihood. Comput Appl Biosci 1997, 13(5):555-556.

17. Nickel GC, Tefft D, Adams MD: Human PAML browser: a database of positive selection on human genes using phylogenetic methods. Nucleic Acids Res 2008, 36 Database: D800-808.

18. Schneider S, Roessli D, Excoffier L: Arlequin: A software for population genetics data analysis. User manual ver 2.000 Genetics and Biometry Laboratory, University of Geneva, Switzerland 2000.

19. Excoffier L, Smouse PE, Quattro JM: Analysis of molecular variance inferred from metric distances among DNA haplotypes: application to human mitochondrial DNA restriction data. Genetics 1992, 131(2):479-491.

20. Stephens M, Smith NJ, Donnelly P: A new statistical method for haplotype reconstruction from population data. Am J Hum Genet 2001, 68(4):978-989.

21. Scheet $P$, Stephens M: Linkage disequilibrium-based quality control for large-scale genetic studies. PLOS Genet 2008, 4(8):e1000147.

22. Bandelt HJ, Forster $P$, Sykes BC, Richards MB: Mitochondrial portraits of human populations using median networks. Genetics 1995, 141(2):743-753.

23. Bandelt $H J$, Forster $P$, Rohl $A$ : Median-joining networks for inferring intraspecific phylogenies. Mol Biol Evol 1999, 16(1):37-48.

24. Rozas J, Sanchez-DelBarrio JC, Messeguer X, Rozas R: DnaSP, DNA polymorphism analyses by the coalescent and other methods. Bioinformatics 2003, 19(18):2496-2497.

25. Schaffner SF, Foo C, Gabriel S, Reich D, Daly MJ, Altshuler D: Calibrating a coalescent simulation of human genome sequence variation. Genome Res 2005, 15(11):1576-1583.

26. Charlesworth B, Morgan MT, Charlesworth D: The effect of deleterious mutations on neutral molecular variation. Genetics 1993, 134(4):1289-1303.

27. Myers S, Bottolo L, Freeman C, McVean G, Donnelly P: A fine-scale map of recombination rates and hotspots across the human genome. Science 2005, 310(5746):321-324.

28. Moreno-Estrada A, Tang K, Sikora M, Marques-Bonet T, Casals F, Navarro A, Calafell F, Bertranpetit J, Stoneking M, Bosch E: Interrogating 11 fastevolving genes for signatures of recent positive selection in worldwide human populations. Mol Biol Evol 2009, 26(10):2285-2297.

29. Sabeti PC, Reich DE, Higgins JM, Levine HZ, Richter DJ, Schaffner SF, Gabriel SB, Platko JV, Patterson NJ, McDonald GJ, et al: Detecting recent positive selection in the human genome from haplotype structure. Nature 2002, 419(6909):832-837.

30. Voight BF, Kudaravalli S, Wen X, Pritchard JK: A map of recent positive selection in the human genome. PLoS Biol 2006, 4(3):e72.

31. Pickrell JK, Coop G, Novembre J, Kudaravalli S, Li JZ, Absher D, Srinivasan BS, Barsh GS, Myers RM, Feldman MW, et al: Signals of recent positive selection in a worldwide sample of human populations. Genome Res 2009, 19(5):826-837.

32. Li JZ, Absher DM, Tang H, Southwick AM, Casto AM, Ramachandran S, Cann HM, Barsh GS, Feldman M, Cavalli-Sforza LL, et al: Worldwide human 
relationships inferred from genome-wide patterns of variation. Science 2008, 319(5866):1100-1104.

33. Nielsen R, Williamson S, Kim Y, Hubisz MJ, Clark AG, Bustamante C: Genomic scans for selective sweeps using SNP data. Genome Res 2005, 15(11):1566-1575.

34. Sabeti PC, Schaffner SF, Fry B, Lohmueller J, Varilly P, Shamovsky O, Palma A, Mikkelsen TS, Altshuler D, Lander ES: Positive natural selection in the human lineage. Science 2006, 312(5780):1614-1620.

35. Frazer KA, Ballinger DG, Cox DR, Hinds DA, Stuve LL, Gibbs RA, Belmont JW, Boudreau A, Hardenbol P, Leal SM, et al: A second generation human haplotype map of over 3.1 million SNPs. Nature 2007, 449(7164):851-861.

36. Reumers J, Conde L, Medina I, Maurer-Stroh S, Van Durme J, Dopazo J, Rousseau F, Schymkowitz J: Joint annotation of coding and non-coding single nucleotide polymorphisms and mutations in the SNPeffect and PupaSuite databases. Nucleic Acids Res 2008, 36 Database: D825-829.

37. Conde L, Vaquerizas JM, Dopazo H, Arbiza L, Reumers J, Rousseau F, Schymkowitz J, Dopazo J: PupaSuite: finding functional single nucleotide polymorphisms for large-scale genotyping purposes. Nucleic Acids Res 2006, , 34 Web Server: W621-625.

38. Anisimova M, Bielawski JP, Yang Z: Accuracy and power of the likelihood ratio test in detecting adaptive molecular evolution. Mol Biol Evol 2001, 18(8):1585-1592.

39. Garcia-Bernal D, Sotillo-Mallo E, Nombela-Arrieta C, Samaniego R, Fukui Y, Stein JV, Teixido J: DOCK2 is required for chemokine-promoted human T lymphocyte adhesion under shear stress mediated by the integrin alpha4beta1.J Immunol 2006, 177(8):5215-5225.

40. Ramirez-Soriano A, Ramos-Onsins SE, Rozas J, Calafell F, Navarro A: Statistical power analysis of neutrality tests under demographic expansions, contractions and bottlenecks with recombination. Genetics 2008, 179(1):555-567.

41. Hruz T, Laule O, Szabo G, Wessendorp F, Bleuler S, Oertle L, Widmayer P, Gruissem W, Zimmermann P: Genevestigator v3: a reference expression database for the meta-analysis of transcriptomes. Adv Bioinformatics 2008, 2008:420747.

42. Kilpinen S, Autio R, Ojala K, lljin K, Bucher E, Sara H, Pisto T, Saarela M, Skotheim Rl, Bjorkman M, et al: Systematic bioinformatic analysis of expression levels of 17,330 human genes across 9,783 samples from 175 types of healthy and pathological tissues. Genome Biol 2008, 9(9):R139.

43. Hancock AM, Witonsky DB, Gordon AS, Eshel G, Pritchard JK, Coop G, Di Rienzo A: Adaptations to climate in candidate genes for common metabolic disorders. PLoS Genet 2008, 4(2):e32.

44. Yang Z: Likelihood ratio tests for detecting positive selection and application to primate lysozyme evolution. Mol Biol Evol 1998, 15(5):568-573.

\section{Submit your next manuscript to BioMed Central and take full advantage of:}

- Convenient online submission

- Thorough peer review

- No space constraints or color figure charges

- Immediate publication on acceptance

- Inclusion in PubMed, CAS, Scopus and Google Scholar

- Research which is freely available for redistribution 